Swarthmore College

Works

$12-1-2005$

\title{
Numerical Modeling Of Hohlraum Radiation Conditions: Spatial And Spectral Variations Due To Sample Position, Beam Pointing, And Hohlraum Geometry
}

\author{
David H. Cohen \\ Swarthmore College, dcohen1@swarthmore.edu \\ O. L. Landen \\ J. J. MacFarlane
}

Follow this and additional works at: https://works.swarthmore.edu/fac-physics

Part of the Astrophysics and Astronomy Commons

Let us know how access to these works benefits you

\section{Recommended Citation}

David H. Cohen, O. L. Landen, and J. J. MacFarlane. (2005). "Numerical Modeling Of Hohlraum Radiation Conditions: Spatial And Spectral Variations Due To Sample Position, Beam Pointing, And Hohlraum Geometry". Physics Of Plasmas. Volume 12, Issue 12. DOI: 10.1063/1.2146863

https://works.swarthmore.edu/fac-physics/12

This work is brought to you for free by Swarthmore College Libraries' Works. It has been accepted for inclusion in Physics \& Astronomy Faculty Works by an authorized administrator of Works. For more information, please contact myworks@swarthmore.edu. 


\section{AIP $\mid$ Physics of Plasmas}

Numerical modeling of Hohlraum radiation conditions: Spatial and spectral variations due to sample position, beam pointing, and Hohlraum geometry

David H. Cohen, Otto L. Landen, and Joseph J. MacFarlane

Citation: Physics of Plasmas (1994-present) 12, 122703 (2005); doi: 10.1063/1.2146863

View online: http://dx.doi.org/10.1063/1.2146863

View Table of Contents: http://scitation.aip.org/content/aip/journal/pop/12/12?ver=pdfcov

Published by the AIP Publishing

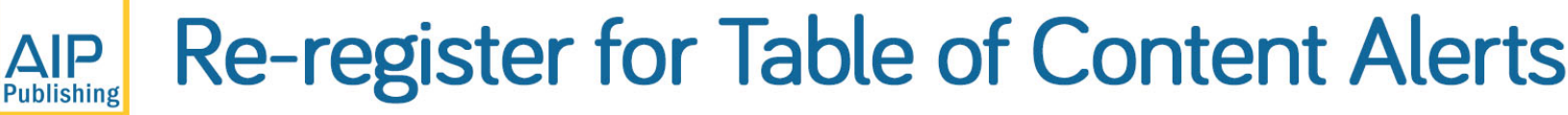




\title{
Numerical modeling of Hohlraum radiation conditions: Spatial and spectral variations due to sample position, beam pointing, and Hohlraum geometry
}

\author{
David H. Cohen \\ Department of Physics and Astronomy, Swarthmore College, Swarthmore, Pennsylvania 19081 \\ Otto L. Landen \\ Lawrence Livermore National Laboratory, Livermore, California 94550 \\ Joseph J. MacFarlane \\ Prism Computational Sciences, 455 Science Drive, Suite 140, Madison, Wisconsin 53711
}

(Received 11 February 2005; accepted 9 November 2005; published online 23 December 2005)

\begin{abstract}
View-factor simulations are presented of the spatially varying radiation conditions inside double-ended gold Hohlraums and single-ended gold Hohlraums ("halfraums") used in inertial confinement fusion and high-energy density physics experiments [J. Lindl, Phys. Plasmas 11, 339 (2004); M. D. Rosen, Phys. Plasmas 3, 1803 (1996)]. It is shown that in many circumstances, the common assumption that the Hohlraum "drive" can be characterized by a single temperature is too simplistic. Specifically, the radiation conditions seen by an experimental package can differ significantly from the wall reemission measured through diagnostic holes or laser entrance holes (LEHs) by absolutely calibrated detectors. Furthermore, even in situations where the radiation temperature is roughly the same for diagnostics and experimental packages, or for packages at different locations, the spectral energy distributions can vary significantly, due to the differing fractions of reemitting wall, laser hot spots, and LEHs seen from different locations. We find that the spatial variation of temperature and especially the differences between what diagnostics looking in the LEH measure versus the radiation temperature on wall-mounted experimental packages are generally greater for double-ended Hohlraums than for halfraums. View-factor simulations can also be used to explore experimental variables (halfraum length and geometry, sample position, and beam pointing) that can be adjusted in order to, for example, maximize the radiation flux onto a sample, or other package. In this vein, simulations of Hohlraums and halfraums with LEH shields are also presented. (C) 2005 American Institute of Physics. [DOI: 10.1063/1.2146863]
\end{abstract}

\section{INTRODUCTION}

Optimizing the time- and wavelength-dependent Hohlraum radiation drive onto a fuel capsule is a key component in achieving ignition in inertial confinement fusion (ICF) experiments. ${ }^{1,2}$ Although much experimental and theoretical effort has been expended in understanding the $\mathrm{x}$-ray drive characteristics of Hohlraums and optimizing the drive symmetry onto the capsule, there have been few studies of the spatial variation of the radiation field conditions within Hohlraums, and especially within halfraums. The x-ray spectrum incident on a surface in a Hohlraum or halfraum, whether part of the wall, a fuel capsule, or some other object within the cylinder, will vary with the location and orientation of the surface according to the relative view factors of wall reemission, laser hot spots, and cold laser entrance holes (LEHs) and diagnostic holes. Detailed view-factor modeling can play an important role in answering questions about this variation and can be used to interpret diagnostics and plan experiments.

In this paper we will present view-factor models of Hohlraums and halfraums, investigating the spatial variations of the radiation field (both overall intensity and spectral energy distribution), and the effects of halfraum size and geometry and of beam pointing. One conclusion from this modeling is that care must be taken in inferring the drive onto an experimental package from a measurement of wall reemission from a particular direction when using an absolutely calibrated detector, such as DANTE. ${ }^{3}$ A more general conclusion is that view-factor simulations are a valuable tool for optimizing the performance of Hohlraum experiments and in interpreting diagnostic measurements.

By their nature, view-factor simulations do not account directly for hydrodynamics, laser-plasma interactions, or detailed atomic physics. View-factor calculations are based on power balance among energy source terms, radiation losses (absorption), and reemission (reflection). On the other hand, view-factor simulations provide a reasonable representation of the radiation field distribution throughout a threedimensional (3D) grid of surfaces, and do so at a small fraction of the CPU costs of multidimensional radiationhydrodynamics codes. View-factor codes can be used effectively in estimating Hohlraum radiation temperatures and predicting the radiation symmetry on ICF capsules. The simulations we present here are relevant to all but the latest times of laser Hohlraum experiments when on-axis stagnation of gold plasma contributes significantly to the radiation properties of a Hohlraum and the associated interpretation of diagnostics. 
We will critically examine the standard analytic treatment of Hohlraum energy balance, in which the radiation properties of a Hohlraum are described by a single "Hohlraum radiation temperature." Although the emission from each computational surface element in our view-factor simulations is taken to be Planckian, the flux incident on any given surface in a simulation (whether wall, target, or diagnostic) can be distinctly non-Planckian. We will show examples where deviations from a blackbody spectrum are nontrivial. We begin by benchmarking DANTE measurements of a Hohlraum experiment ${ }^{4}$ on OMEGA. ${ }^{5}$ We then show that experimental packages can be subject to radiation conditions that are quite different than those seen by DANTE, even when that diagnostic is used on an optimal LEH-viewing line of sight.

The focus of this study will be on effectively empty Hohlraums and halfraums. However, we do investigate how the radiation conditions change with the presence of a capsule in Sec. III. In Sec. IV we explore the fundamental differences between Hohlraums and halfraums, in terms of both DANTE measurements and the radiation onto an experimental package. (Note that we use the terms "sample" and "package" interchangeably in this paper.) In Sec. V we show how variations in the beam pointing and LEH size affect the radiation conditions and DANTE measurements. In Sec. VI we explore the effects of halfraum length and the presence of foils and shields.

\section{SPATIAL DEPENDENCE OF THE RADIATION DRIVE IN A HOHLRAUM}

We first present the results of simulations of a set of OMEGA experiments reported on by Decker et al. ${ }^{4}$ In these experiments, the Hohlraum temperature was inferred by measuring the absolute flux emitted from a portion of the Hohlraum wall. Ten $42^{\circ}$ OMEGA beams (cone 2 ) and twenty $59^{\circ}$ OMEGA beams (cone 3), having an energy of $500 \mathrm{~J}$ each and with $1 \mathrm{~ns}$ square profiles, illuminated a standard (2300 $\mu \mathrm{m}$ length $\times 1600 \mu \mathrm{m}$ diameter) gold Hohlraum, with three-quarter (or $1200 \mu \mathrm{m}$ diameter) LEHs. Threequarter LEHs are used in all the models we present in this paper, unless otherwise noted. The beam pointing in these experiments and in our modeling was such that all 15 beams on each side of the Hohlraum made a single ring of hot spots, centered $480 \mu \mathrm{m}$ from the LEH plane. The beams were all focused at the pointing spot, where they crossed the long Hohlraum axis (at the LEH plane for the cone 3 beams and $400 \mu \mathrm{m}$ outside of the Hohlraum for the cone 2 beams).

One purpose of the experimental campaign of Decker et al. was to show that the absolutely calibrated x-ray detector, DANTE, gives a better indication of the Hohlraum radiation conditions seen by a capsule when it views the wall reemission through the $\mathrm{LEH}$, rather than through a diagnostic hole at the midplane. The Hohlraums in these experiments were on the P5-P8 axis of the OMEGA target chamber, so that the DANTE viewing angle was $37.4^{\circ}$ with respect to the Hohlraum axis (see Fig. 1 for a model of the Hohlraum target, including the DANTE view of this configuration).

We performed a series of simulations of these Hohlraum
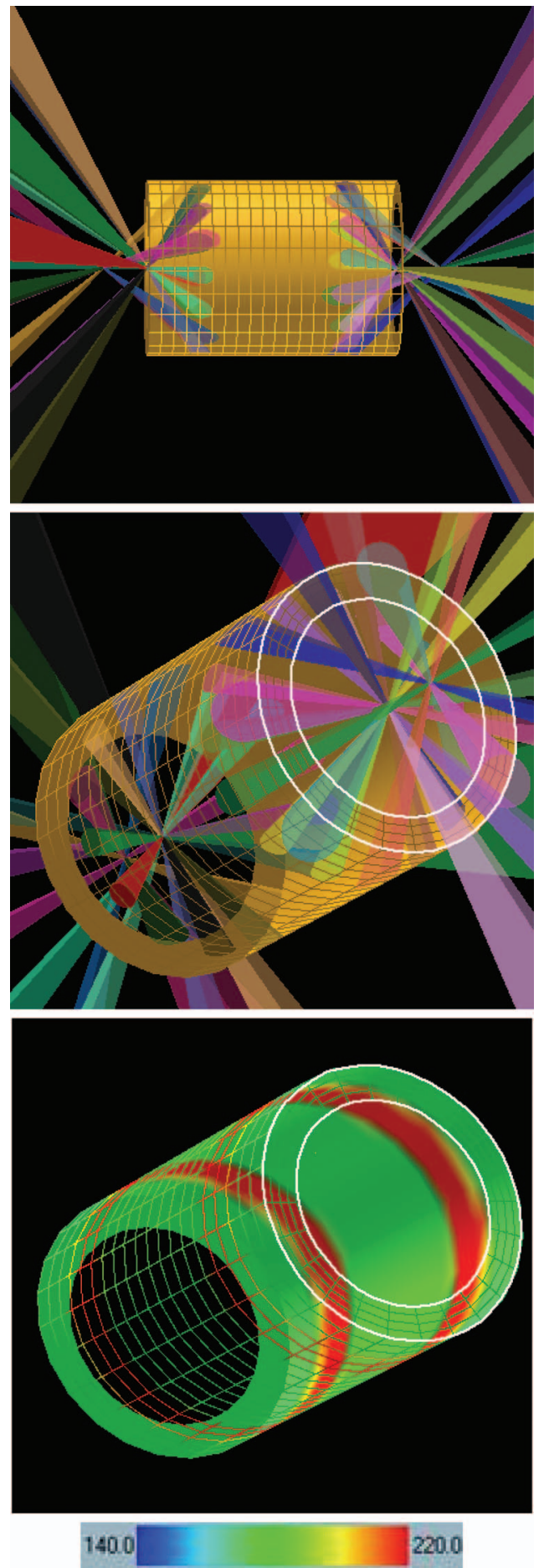

FIG. 1. (Color) Hohlraum images generated with the VISRAD view-factor code, relevant to the experiments discussed in Ref. 4. The top panel shows the OMEGA beam pointing into the Hohlraum cylinder seen side on. Note that the cone 2 beams on each side are pulled back so that the beams from both cones make a single ring on each side of the Hohlraum. The middle panel shows the same target model, but from the position of the DANTE diagnostic. The lower panel shows the DANTE view again, but with the beams hidden, and with the wall temperature at $t=1.0 \mathrm{~ns}$ displayed as a color map (the dynamic range in this, and all other, temperature color maps shown in the paper is $140-220 \mathrm{eV}$ ). Note the ring of laser hot spots on each side of the Hohlraum. Note also in all of these images how structures in the model seen from the back, or outside, are rendered as transparent mesh to allow for an unobstructed view of the interior of the Hohlraum. This convention will be used throughout the paper. Finally, we point out that the "front," DANTE-facing, LEH is in the upper right in this, and all similar figures throughout the paper, and the inner and outer edges of the front LEH lip are highlighted in white. 


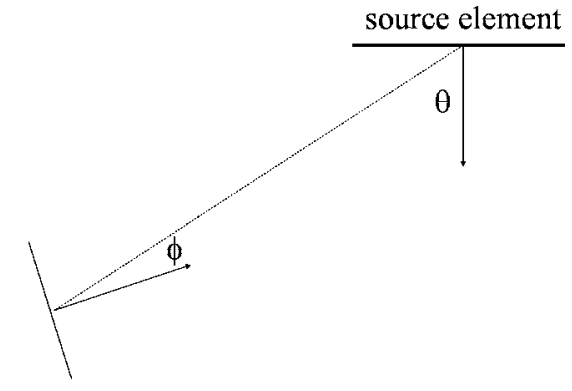

surface element

FIG. 2. Schematic of the view-factor calculation for an arbitrary geometry. The flux from any source element onto any other surface element is proportional to the cosine of the angle between the line of centers of the two elements and the surface normal of the source element (because the source element is assumed to be a Lambertian emitter) and also to the cosine of the line of centers and the normal of the surface element (accounting for the projected cross section of the surface element as seen by the source). The line of centers is indicated by the dashed line while the two surface normals are indicated by arrows.

experiments on OMEGA using the VISRAD 3D view-factor code (v3.1). ${ }^{6}$ VISRAD computes the spatially dependent radiation flux about a 3D grid of surface elements using a steadystate power balance model and material-dependent reflection fractions (albedos). Each surface element is treated as a spatially thin, optically thick Lambertian source with a Planckian frequency dependence. Thus, the (non-Planckian) spectrum incident on a given surface element is composed of contributions from multiple Planckian sources. These contributions to the radiation flux incident on each surface element are summed for each surface element over all other source elements, accounting for the solid angle of the source as seen from the sample, as well as the incident angle of the source radiation onto the sample. See Fig. 2 for a schematic showing these geometrical considerations. Note that the Lambertian flux on the sample is proportional to $\cos \theta \cos \phi$.

Laser beam energy deposition is computed using realistic space and time profiles for the beams (including the $f / 6.7$ beam effective focal ratio of OMEGA), in conjunction with 3D ray-trace algorithms for determining beam-target intersections. The 3D ray-trace algorithm, in which each laser beam is subdivided into a large number of "beamlets," is used to determine which surface elements are hit by a given portion of a laser beam. While VISRAD also computes the specular reflection of laser light off surfaces (glint), laser reflectivities for all surfaces were assumed to be zero for the simulations described here. The target components in the calculation are modeled as a discretized grid of surface elements. The time-dependent albedo and $\mathrm{X}$-ray conversion efficiency (XCE) are input parameters. In the simulations discussed below, the albedo, which is assumed to be spatially uniform, is based on one-dimensional (1D) radiationhydrodynamics simulations of a gold foil exposed to a radiation drive consistent with that in the OMEGA experiments.

We note that the view-factor modeling ignores hydrodynamic motion of the Hohlraum walls, as well as nonequilibrium effects, internal energy stored in the walls by the radiative heating of the object elements, and detailed opacities and emissivities for the Hohlraum. This modeling also ne- glects the effect of temperature gradients in the Hohlraum walls, which can lead to a viewing-angle dependence of emission temperature. We stress that view-factor codes play a complementary role to atomic and hydrodynamics codes. Our goal here is not to calculate wall motion nor the detailed atomic physics and associated line spectra. However, the view-factor modeling accounts for the spatial variation of the radiation conditions, and to some extent, the generally nonPlanckian spectra in Hohlraum environments (via the summation over numerous blackbody surface elements of different temperatures). The time variations of the Hohlraum radiation properties in a VISRAD simulation are computed by making a series of steady-state calculations, each using appropriate beam powers, $\mathrm{x}$-ray conversion efficiencies, and albedos.

To model the OMEGA experiments described above, we calculated the radiation onto a surface at the position of the DANTE diagnostic every 100 ps, using the beam and Hohlraum properties described at the beginning of this section. We assumed perfect square pulses with exactly $1.0 \mathrm{~ns}$ duration and $500 \mathrm{~J}$ total energy per beam. We incorporated a simple model of the laser $x$-ray conversion efficiency, with a linear ramp up to a value of 0.55 at $200 \mathrm{ps}$, and a constant value thereafter. Here, the $\mathrm{x}$-ray conversion efficiency refers to the fraction of incident laser power that is converted to $\mathrm{x}$-ray radiation. The remainder of this energy is converted into kinetic or internal energy or can be lost to the system via laser scattering. In our view-factor calculations, the partitioning of this nonradiative energy is not modeled. We note that the actual value of the XCE does not affect the spatial variation of the temperature at all. The product of XCE and laser power is the relevant quantity, so a change in the XCE is no different than a corresponding change in the laser power, effectively scaling the temperature up or down, in a spatially uniform way.

For the gold albedo, we use the results of a 1D, timedependent hydrodynamics simulation of $\mathrm{x}$-ray deposition onto an infinite gold slab. ${ }^{7}$ We use a detailed opacity model for the gold and monitor the reemission of $\mathrm{x}$ rays by the irradiated gold slab. The albedo is simply the ratio of the reemitted to incident $\mathrm{x}$-ray flux. The albedo value peaks at 0.73 at $1 \mathrm{~ns}$, for a constant power laser pulse, generating a rising x-ray drive, reaching approximately $190 \mathrm{eV}$. We assume that the time-dependent gold albedo is spatially uniform. Detailed radiation diffusion calculations show that this approximation is acceptable, especially for square laser pulses and at late times, ${ }^{8}$ which are most relevant to the simulations we present here.

In Fig. 3 we show the assumed XCE and calculated albedo along with the modeled DANTE temperatures and the DANTE data obtained by Decker et $a l^{4}$ Note that our modeling reproduces the observed DANTE data at all times well within the $6 \%$ error bars on the data. These simulations do not include a capsule, in order to facilitate comparisons to an equivalent halfraum. In the next section we show that the presence of a capsule simply lowers the calculated radiation temperature and does so quite uniformly, so that there is effectively a degeneracy between the presence of a capsule and an increase in the XCE. 

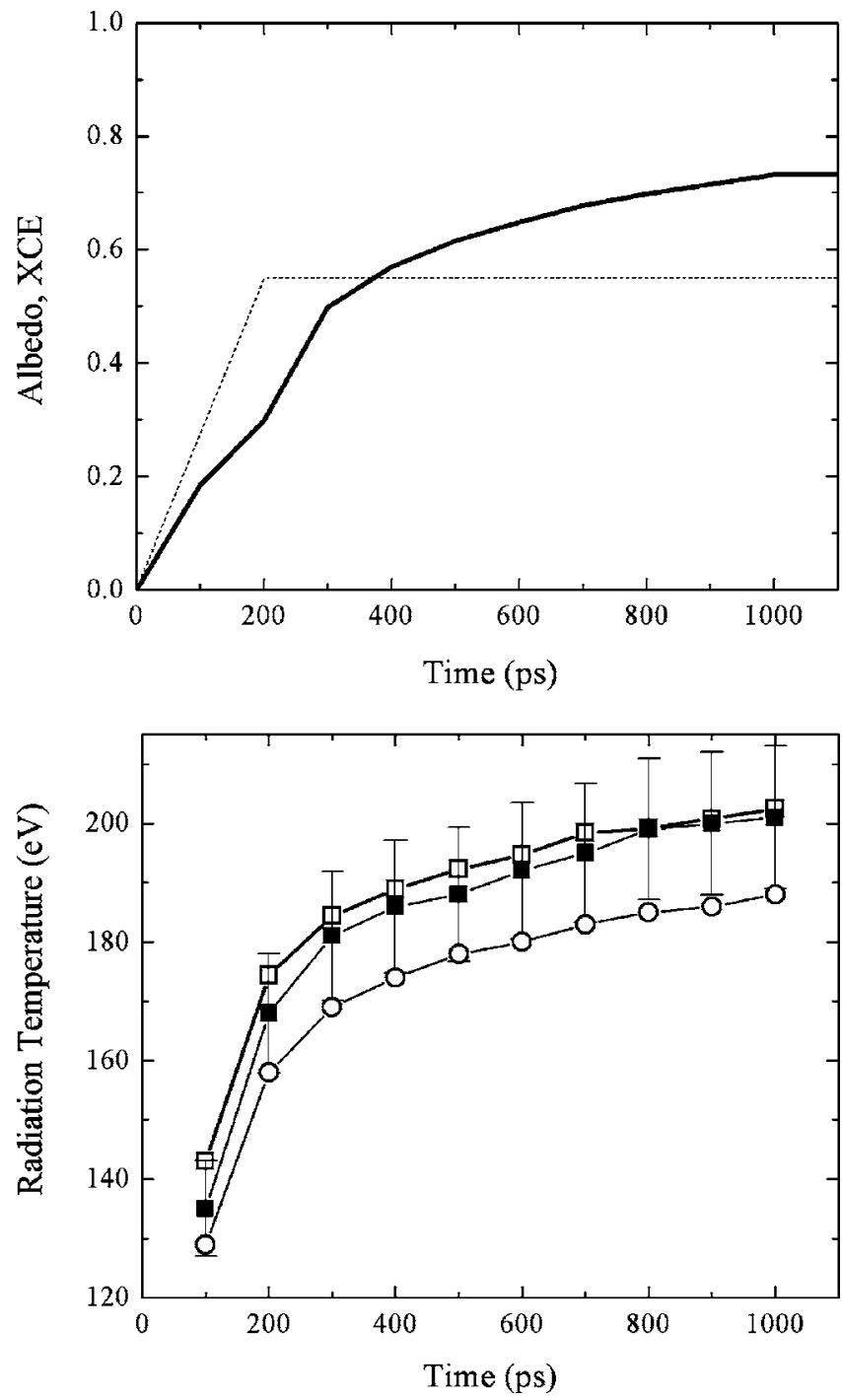

FIG. 3. The top panel shows the assumed $\mathrm{x}$-ray conversion efficiency (dashed line) and calculated albedo (solid line), used as inputs to the viewfactor simulations, the results of which are shown in the lower panel. In the lower panel, the filled squares with error bars are the DANTE temperature measurements from Ref. 4 while the open squares are the simulated DANTE temperatures from the view-factor calculations. The circles are the simulated radiation temperatures at the midplane wall of the Hohlraum.

In Fig. 3 we also show the time-dependent incident radiation temperature on the Hohlraum wall at the midplane, where an experimental package might be mounted. It is significantly $(\sim 15 \mathrm{eV})$ lower than the DANTE temperature. This is due to the less favorable view factor of laser hot spots from the midplane wall compared to DANTE, but mainly due to the contribution from the cold LEHs. In various Hohlraum experiments, some type of sample or other package is placed at the Hohlraum midplane, to expose it to the radiation drive. Historically, Hohlraum radiation conditions have also been diagnosed from midplane wall reemission flux, ${ }^{9-11}$ which is equal to the midplane wall incident radiation multiplied by the albedo. Clearly, Hohlraum radiation diagnostic results will vary depending on the location of the wall reemission they sample. Thus, while DANTE measurements through the LEH provide valuable data on the Hohlraum radiation characteristics, they do not provide a

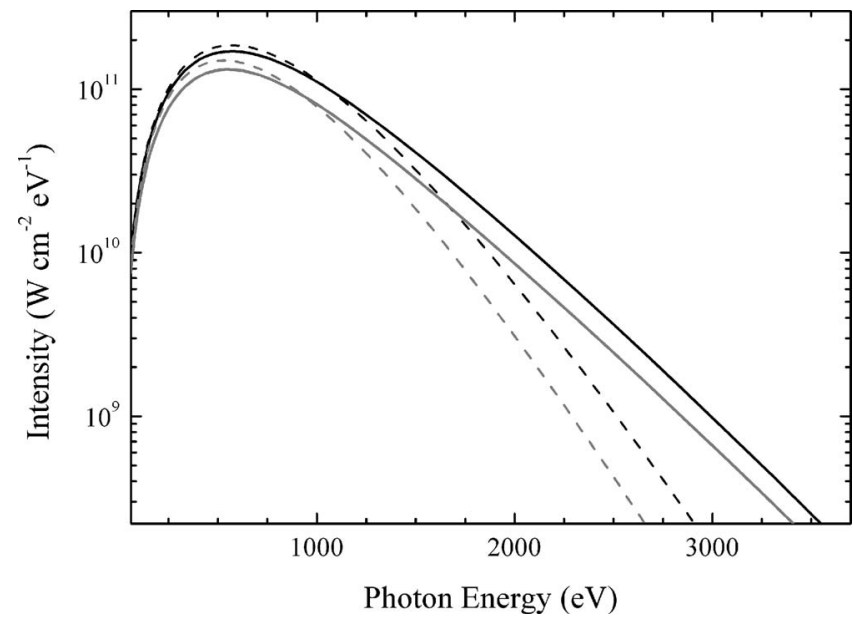

FIG. 4. The simulated DANTE spectrum (solid black) along with the equivalent blackbody spectrum (dashed black) for $t=1.0 \mathrm{~ns}$ in the VISRAD Hohlraum simulation and the simulated spectrum incident on the Hohlraum wall at the midplane (solid gray) along with its equivalent blackbody spectrum (dashed gray) from the same simulation time. Note that the radiation temperatures are $202 \mathrm{eV}$ for DANTE and $188 \mathrm{eV}$ for the midplane wall.

direct measure of the radiation field seen by an experimental package. The differences between the wall temperatures seen by DANTE and the radiation drive temperatures seen by a package can be $\sim 7 \%-8 \%$. This means that the radiation flux $\left(F_{\text {rad }}=\int_{0}^{\infty} F_{\nu} d \nu=\sigma T_{R}^{4}\right.$, where $\sigma$ is the Stefan-Boltzmann constant; note that "radiation temperature," $T_{R}$, is defined by this equation) and therefore the energy absorbed by the experimental package can be different by $\sim 30 \%-35 \%$ compared to what would be inferred using the DANTE measurement directly.

It is useful to compare these detailed calculations to the simpler and more traditional analytic power balance treatment. We use $\eta P_{L}=P_{\text {rad }}=\left[(1-\alpha) A_{\text {wall }}+A_{\mathrm{LEH}}\right] \sigma T_{R}^{4}[\mathrm{see}$, for example, Eq. (1) in Ref. 9], where $P_{L}$ is the laser power, $\eta$ is the XCE, $P_{\text {rad }}$ is the X-ray power, $A_{\text {wall }}$ and $A_{\mathrm{LEH}}$ are the areas of the wall and LEH, respectively, and $\alpha$ is the albedo.

Using a value of $\eta=0.55$ for the XCE, we find at $t=1.0 \mathrm{~ns}$ when the albedo is $\alpha=0.73$, a value of $T_{R}=192 \mathrm{eV}$ for the "Hohlraum radiation temperature." This, as expected, is somewhat less than the DANTE temperature, both in our modeling $(202 \mathrm{eV})$ and in the experiments $(201 \mathrm{eV})$, since DANTE, unlike any point inside the Hohlraum, does not see any of the cold LEH regions. It is very close to the radiation temperature on the sample (191 eV for a wall-facing sample at the center of the Hohlraum, $190 \mathrm{eV}$ for a LEH-facing sample, and $188 \mathrm{eV}$ for a wall-mounted sample), as expected. Note that increasing the XCE to $\eta=0.69$ gives an analytic Hohlraum temperature that agrees with the DANTE temperature at $t=1.0 \mathrm{~ns}$. This higher value of the XCE is in better agreement with the values that are usually assumed. ${ }^{9}$

It is interesting also to compare the spectral energy distribution of the radiation incident on the midplane to that measured by DANTE. In Fig. 4 we show the simulated DANTE spectrum at $t=1.0 \mathrm{~ns}$ along with that incident on the midplane Hohlraum wall. For reference, we also show the equivalent blackbody spectra (the Planckian spectra having 
the same integrated power, or radiation temperature, as the calculated spectra). Note that both spectra are harder than the equivalent blackbody spectra, and that this effect is somewhat stronger for the midplane, where the significant view factor of cold LEHs leads to a deficit of low-energy photons.

Of course, the differences between the DANTE (through the LEH) and midplane radiation conditions will depend on beam pointing. In general, the farther in the pointing, the stronger the radiation will be at the Hohlraum midplane. This is due both to the hot spots being closer to the midplane and to less radiation escaping out of the LEHs. The situation for the DANTE looking in the LEH is more complicated and depends on the relative fraction of the sky occupied by laser hot spots, as seen from DANTE's position. To investigate this, we performed two additional simulations, identical to the one presented above, except for the beam pointing. In the first variation, the ten cone 2 beams are pointed $400 \mu \mathrm{m}$ farther into the Hohlraum, giving a mean laser spot position of $620 \mu \mathrm{m}$ from the LEH plane (we refer to this pointing as "nominal" throughout this paper). Like the cone 3 beams, they are pointed at the center of the LEH, which creates a second ring of five hot spots on either side of the Hohlraum, closer to the midplane than the single ring in the initial simulations, which have a mean spot position of $480 \mu \mathrm{m}$ from the LEH plane. In the second variation, all 30 beams are pointed an additional $200 \mu \mathrm{m}$ farther into the Hohlraum, giving a mean spot position of $820 \mu \mathrm{m}$ from the LEH plane.

In Fig. 5 we show the results of this series of simulations with varying beam pointings. The radiation drive temperature onto the midplane wall does, in fact, increase as the beam pointing moves farther in the Hohlraum toward the midplane. The DANTE temperature increases almost as much, but for a different reason, as the pointing moves inward and hence out of the partial occlusion of the lip. As in the original simulations, these Hohlraums' LEHs have 75\% of the Hohlraum diameter and thus have annular lips. The lip certainly can affect the DANTE view of hot spots and wall reemission, which we explore in the context of halfraums in Sec. V. In closing, we note that the trends shown here are very similar when we look at earlier times, where both the albedo and the XCE are lower than they are at $1 \mathrm{~ns}$. The only notable difference is the greater similarity between the DANTE and sample temperatures for the deepest pointing at early times, as can be seen in the lower panel of Fig. 5 .

\section{EFFECTS OF A CAPSULE}

A spherical fuel capsule located at the center of the Hohlraum acts as another sink of photons (due to the relatively low albedo of the plastic capsule) and will lower the Hohlraum temperature accordingly. To explore the nature and magnitude of this effect, we repeated the analytic Hohlraum radiation temperature calculation with a 520- $\mu$ m-diam spherical capsule in the Hohlraum, and then we repeated two of the view-factor simulations discussed above, but with a capsule. We assumed a capsule albedo of $\alpha=0.3$ independent of time ${ }^{12}$ and also assumed that the capsule size remained constant over the $1.0 \mathrm{~ns}$ duration of the simulation.
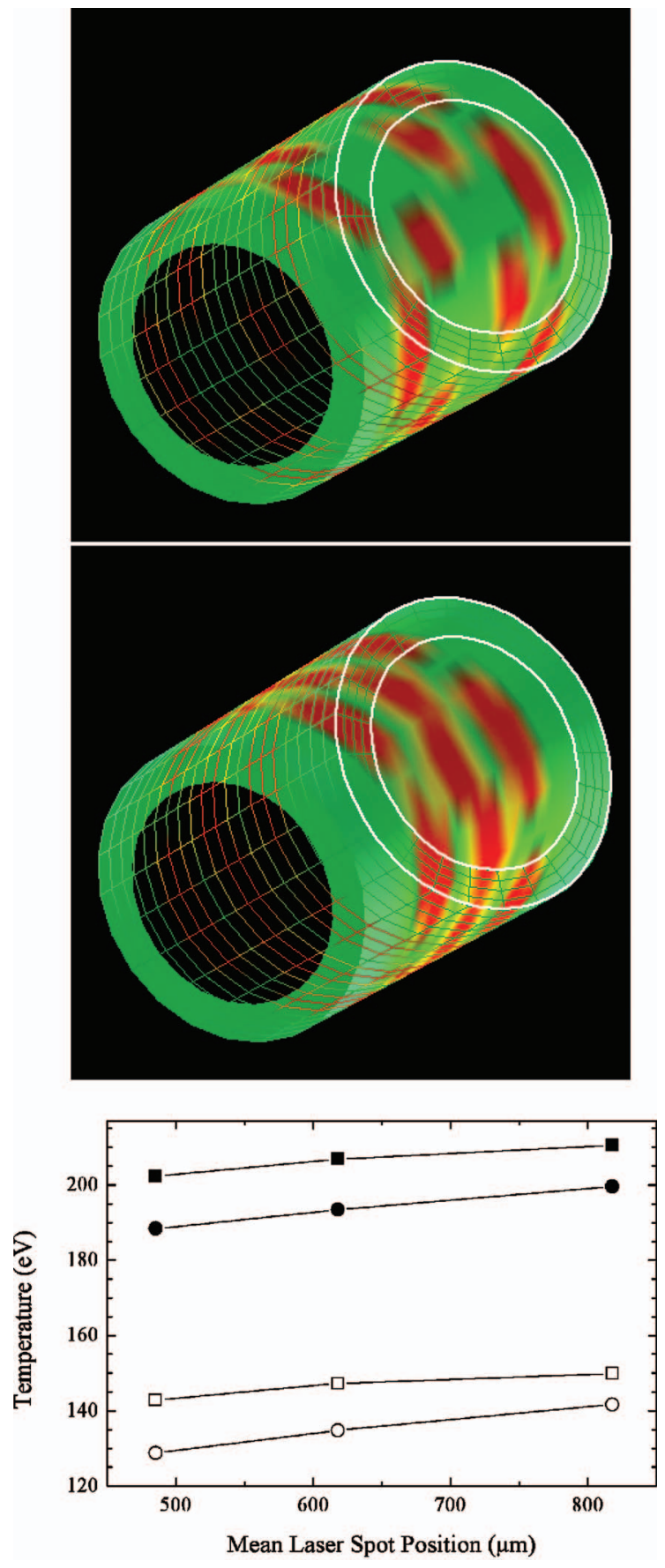

FIG. 5. (Color) The DANTE views of the Hohlraum in the two cases with different beam pointings (top two panels). As in the bottom panel of Fig. 1, we show a color map of emission temperature at $t=1.0 \mathrm{~ns}$, and hide the beams for clarity. The color scale spans from 140 to $220 \mathrm{eV}$. The lower panel shows the trends of DANTE temperature (squares) and midplane temperature (circles) as the beam pointing is changed. The filled symbols represent the simulation time $t=1.0 \mathrm{~ns}(\mathrm{XCE}=0.55$ and albedo $=0.73)$ and the open symbols represent the simulation time $t=100 \mathrm{ps} \quad(\mathrm{XCE}=0.28$ and albedo $=0.18$ ). The original model, used to reproduce the experiments reported on in Ref. 4 has a mean laser spot position of $480 \mu \mathrm{m}$ from the LEH plane. The first variation $(620 \mu \mathrm{m})$ is shown in the top panel and the second variation $(820 \mu \mathrm{m})$ is shown in the middle panel. We note that in this last case, the cone 2 beams from either side of the Hohlraum hit the wall almost exactly at the midplane, creating a single, combined ring of hot spots.

The Hohlraum temperature calculation discussed in the previous section was modified to include the area of the capsule: $\eta P_{L}=P_{\text {rad }}=\left[(1-\alpha) A_{\text {wall }}+A_{\mathrm{LEH}}+\left(1-\alpha_{\text {cap }}\right) A_{\text {cap }}\right] \sigma T_{R}^{4}$. To calculate the Hohlraum radiation temperature at $t=1.0 \mathrm{~ns}$, we used $\eta=0.55$ for the XCE and $\alpha=0.73$, as we did previ- 

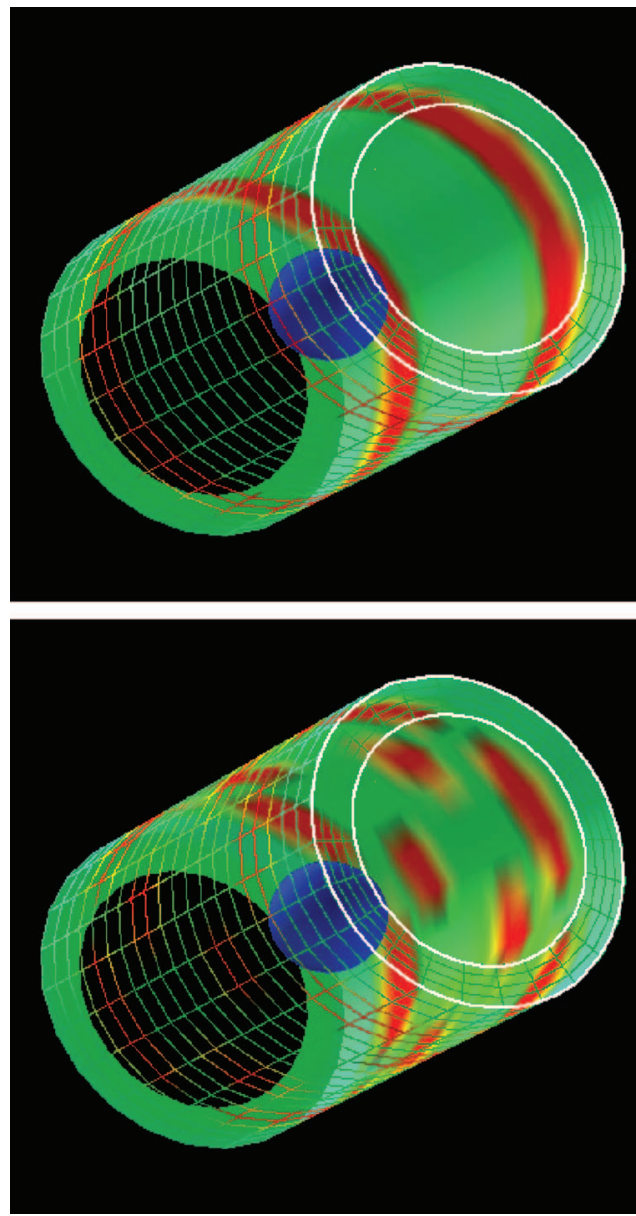

FIG. 6. (Color) VISRAD simulations of Hohlraums with fuel capsules. The capsules are centered in the Hohlraums and have a diameter of $520 \mu \mathrm{m}$ and an albedo of $\alpha=0.3$. The top panel shows the "single ring" pointing (compare to the bottom panel in Fig. 1) while the bottom panel shows the "nominal" pointing (compare to the top panel in Fig. 5) in which two rings are formed by pointing both the cone 2 and cone 3 beams at the LEH center.

ously, while the capsule albedo was assigned a value of $\alpha_{\text {cap }}=0.3$. For these values, we find that the capsule's presence lowers the Hohlraum radiation temperature by $4 \mathrm{eV}$ to $T_{R}=188 \mathrm{eV}$ at $t=1.0 \mathrm{~ns}$.

We repeated two of the numerical view-factor simulations: one with the "single ring" pointing used in the experiments of Decker et al. (corresponding to Fig. 1) and the other with the "nominal" pointing in which all the beams are pointed at the middle of the LEH (corresponding to the upper panel of Fig. 5). In Fig. 6 we show the results of these two new simulations, which do, indeed, show lower radiation temperatures than the corresponding simulations without capsules.

In Fig. 7 we compare the time-dependent radiation temperature from each simulation. At $t=1.0 \mathrm{~ns}$, the DANTE temperature is $4 \mathrm{eV}$ lower with the capsule in the case of the nominal pointing (202 eV vs $206 \mathrm{eV}$ ) and $6 \mathrm{eV}$ lower for the single ring pointing (196 eV vs $202 \mathrm{eV}$ ), in good agreement with the analytic power balance calculation. The sample temperature drop is $7 \mathrm{eV}$ in the case of the nominal pointing $(186 \mathrm{eV}$ vs $193 \mathrm{eV})$ at $t=1.0 \mathrm{~ns}$ and $6 \mathrm{eV}$ lower in the case of the single ring pointing (182 eV vs $188 \mathrm{eV})$. The slightly
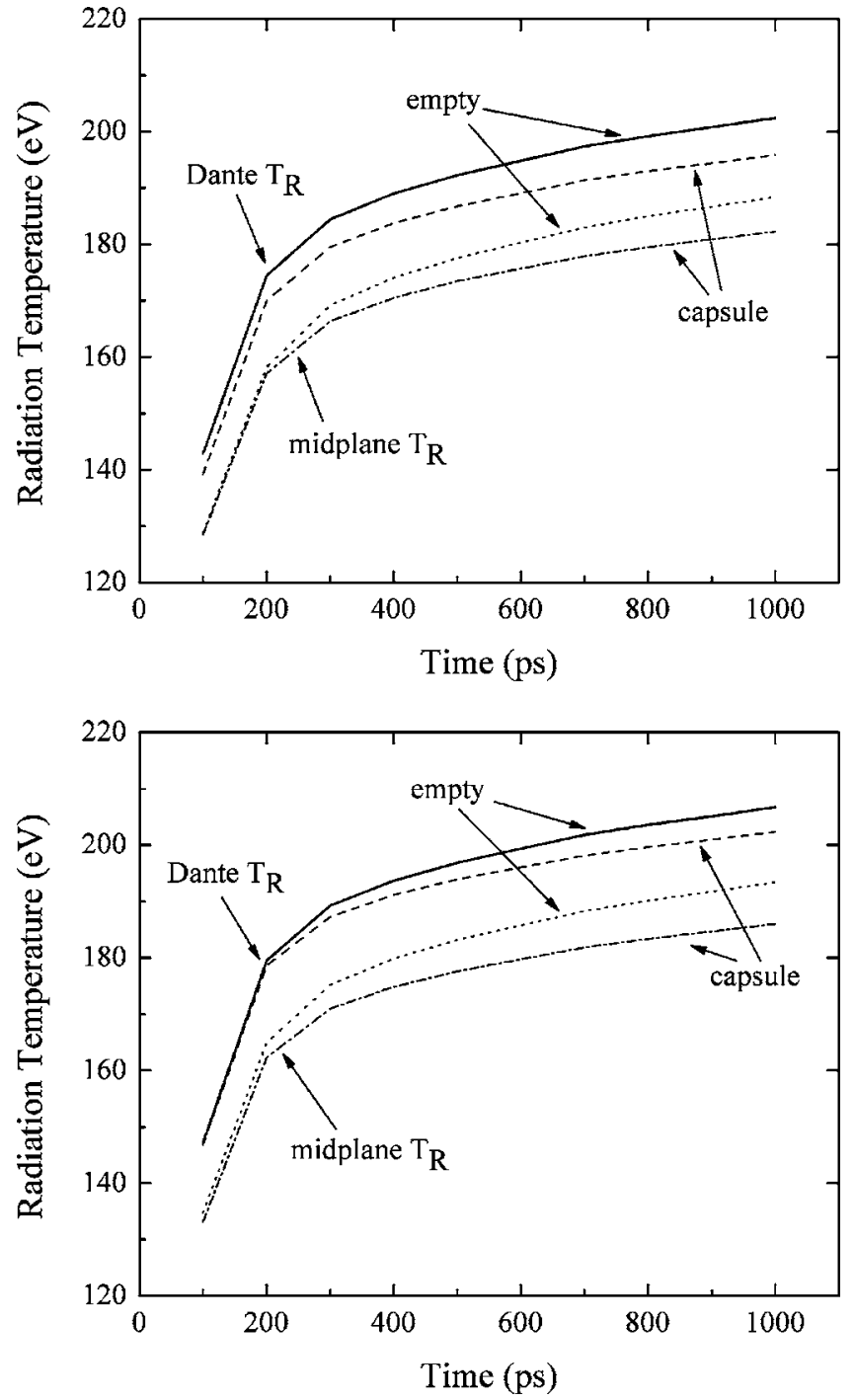

FIG. 7. Calculated radiation temperature vs time for the VISRAD simulations shown in Fig. 6. The top panel is for the "single ring" pointing while the bottom panel is for the "nominal" pointing. In each panel, we show the DANTE temperature for the empty (i.e., no capsule) Hohlraum as a solid line and for the Hohlraum containing a capsule as a dashed line. The radiation temperature seen by a wall-mounted sample at the midplane is denoted by a dotted line for the empty Hohlraum and by a dash-dot line for the Hohlraum with a capsule.

larger temperature decreases due to the presence of the capsule in the case of the wall-mounted samples arises from the large view factor of the capsule as seen by the sample, which is mounted at the midplane wall, and thus is quite close to the capsule. In the case of the nominal pointing in which the capsule's presence has a bigger effect on the sample temperature, the capsule's blocking of some of the laser hot spots as seen from the sample's position plays a role too.

We can also see in Fig. 7 that the effect of the capsule on the calculated radiation temperatures is less extreme at earlier times, when the capsule albedo and the Hohlraum wall albedo have more similar values. But other than these small differences in the DANTE versus sample radiation temperature and in the time dependence of the effect of the capsule, the capsule's effect is a uniform lowering of the radiation temperature in the Hohlraum that corresponds to roughly 
$2.5 \%$ or roughly $10 \%$ in radiation flux. By increasing the converted laser power by a corresponding $10 \%$, the results of the simulations without the capsule are reproduced with the capsule. This increase in power corresponds to the increase of the XCE from 0.55 to 0.60 . Thus the results of the viewfactor simulations discussed in the remainder of this paper are applicable to Hohlraums with capsules and slightly higher XCEs, although in detail, the temporal, spatial, and spectral dependencies will be affected by a capsule. If a high degree of accuracy is required in analyzing these variations, then detailed simulations should be performed.

\section{EVOLUTION TO A HALFRAUM}

Increasingly, indirect drive and related experiments are performed in halfraums, ${ }^{13,14}$ which are shorter cylinders with only one LEH, sometimes referred to as half Hohlraums, or single-ended Hohlraums. Experimental packages in halfraums are often mounted on the end of the cylinder, opposite to the LEH. We might expect to see similar effects to those we demonstrated in the previous sections: spatial dependence of the drive properties within a halfraum (both in terms of overall power and in terms of the spectral energy distribution) and, specifically, differences between DANTE measurements and the radiation drive incident upon an experimental package.

Because a halfraum is essentially just half of a Hohlraum, one expects its properties to not differ appreciably from those of a Hohlraum. There are only half as many beams in a halfraum, but the LEH area is exactly half of that in a Hohlraum, and the wall area is approximately half as large. One difference between a Hohlraum and a halfraum, for experiments with packages that are planar samples, is that a sample located at the midplane of a Hohlraum is typically mounted on the wall, or barrel, of the Hohlraum, facing the opposite wall. In a halfraum, a planar package is typically on the back end of the halfraum, facing the LEH. So there is a difference in the position and orientation of the sample, which will affect the relative view factors of hot spots and LEH, as compared to the case of a planar sample mounted at the midplane of a Hohlraum. In order to investigate the effect of sample position and orientation, we first repeated our initial Hohlraum simulations (without a capsule and with the simple single ring beam pointing such that both cone 2 and cone 3 beams make a single ring of hot spots $480 \mu \mathrm{m}$ from the LEH plane), but we located the planar sample in the middle of the Hohlraum, suspended where a capsule would be. We performed four such simulations, varying only the sample orientation from wall facing to LEH facing. The results of these four simulations are shown in Fig. 8.

We find that the radiation temperature onto a planar sample at the center of the Hohlraum is almost completely independent of sample orientation at $t=1.0 \mathrm{~ns}$, when the albedo is high $(\alpha=0.73)$. It is also nearly identical to the radiation temperature on a wall-mounted planar sample at the midplane. This result is relevant for experiments that, for example, investigate shock timing on wall-mounted packages and use the results to infer the drive onto a capsule.

The variation among these five cases (four at the center

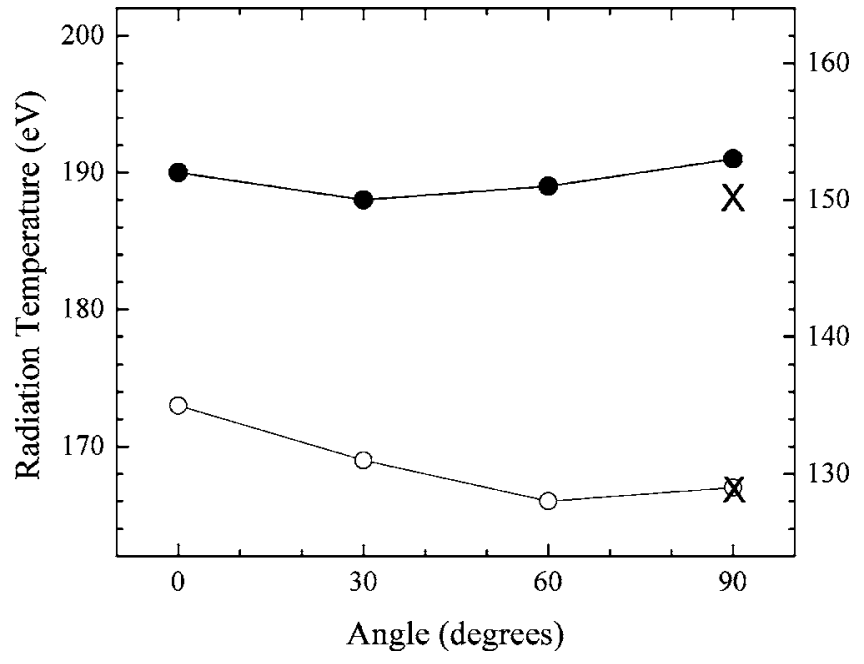

FIG. 8. Radiation temperature as a function of sample orientation for a planar sample located at the center of a Hohlraum. The angle plotted along the $x$ axis is the angle between the sample normal and the Hohlraum axis, so that $0^{\circ}$ is LEH facing, while $90^{\circ}$ is wall facing. The filled symbols are the results from $t=1.0 \mathrm{~ns}$, while the open symbols are from $t=100 \mathrm{ps}$. For comparison, the radiation temperatures at these two times for a sample on the wall of the Hohlraum at the midplane (discussed in Sec. II) are 188 and $129 \mathrm{eV}$ (denoted by Xs); nearly identical to the centrally located, wallfacing $\left(90^{\circ}\right)$ results shown here. Finally, we note that the DANTE temperatures for these two times are 202 and $143 \mathrm{eV}$, respectively. In this, and all other figures showing temperature trends in the remainder of the paper, the left-hand axis refers to the values at $t=1 \mathrm{~ns}$ (solid symbols), while the righthand axis refers to the values at $t=100 \mathrm{ps}$ (open symbols).

of the Hohlraum and one on the wall) is only $3 \mathrm{eV}$ at $t=1.0 \mathrm{~ns}$, with no monotonic trend with orientation. Indeed, this result simply reflects the fact that cylindrical Hohlraums, and their associated laser heating schemes, have been designed to generate a nearly uniform radiation drive onto a fuel capsule at their centers. The view factors of hot spots and cold LEHs change in concert with each other as the sample orientation changes. However, although the radiation temperature is nearly independent of sample orientation, the spectral energy distribution is not. In Fig. 9 we compare the spectrum incident upon a sample facing the LEH with that incident on the same sample facing the Hohlraum wall. The radiation temperatures in these two cases are nearly identical $(190 \mathrm{eV}$ vs $191 \mathrm{eV})$, but the LEH-facing sample has a significantly harder spectrum than the wall-facing sample (>25\% more flux at $2 \mathrm{keV}$; and if the gold $M$ band were explicitly taken into account by the modeling, this difference could be larger). This is because the LEH-facing sample has more high-energy radiation incident upon it due to its larger hot-spot view factor and less lower-energy wall radiation due to its larger LEH view factor.

We also note, referring to Fig. 8, that there is a somewhat larger dependence of radiation temperature on sample orientation at early times, when the albedo is lower $(\alpha=0.18$ at $t=100 \mathrm{ps})$ than at late times. The radiation temperature is $6 \mathrm{eV}$ higher for the LEH-facing sample than for the wall-facing sample at $t=100 \mathrm{ps}$. This is due to the fact that the wall-facing sample's view is dominated by a significantly cooler wall in a low-albedo situation.

Finally, it has been noticed that the DANTE temperature 


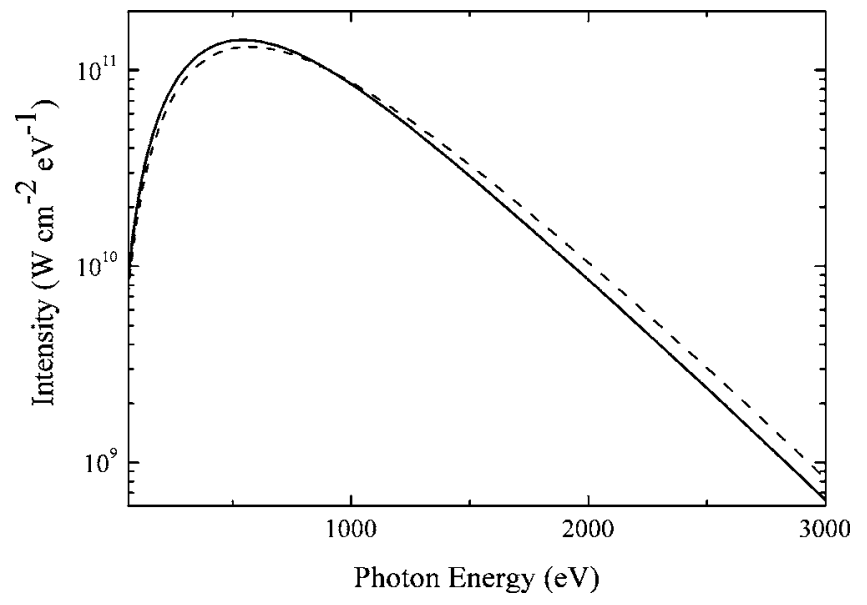

FIG. 9. Comparison of the spectrum incident on a sample at the center of a Hohlraum (at $t=1.0 \mathrm{~ns}$ ) when it is oriented toward the LEH (dashed line, $0^{\circ}$ case in the previous figure) vs the spectrum when the sample is oriented toward the Hohlraum wall (solid line, $90^{\circ}$ case in the previous figure). The LEH-facing sample has a modestly harder spectrum, though the radiation temperature onto each is nearly identical (190 eV vs $191 \mathrm{eV})$.

more closely tracks the sample temperature in a halfraum configuration than in a similar Hohlraum configuration. ${ }^{14}$ Based on the above analysis, we see that this is not due to the difference in the sample position or orientation as one goes from a Hohlraum to a halfraum. The sample radiation temperature does not change significantly as the sample is moved from the midplane Hohlraum wall to the center of the Hohlraum and turned to face the LEH. In order to ascertain what accounts for the better agreement between the DANTE temperature and the sample temperature in the halfraum (recall that this difference is about $15 \mathrm{eV}$ in a Hohlraum), we constructed a model of a halfraum by simply taking our Hohlraum model having the sample in the center of the volume and facing the LEH, and inserting a gold disk at the midplane, to effectively divide the Hohlraum in half, giving the resulting halfraum a length of $1150 \mu \mathrm{m}$. The DANTE view of the halfraum is shown in Fig. 10, which may be compared with the view of the corresponding Hohlraum in Fig. 1(c).

In the Hohlraum case, the DANTE temperature is $202 \mathrm{eV}$ and the sample temperature is $190 \mathrm{eV}$ (difference of $28 \%$ in flux). In the halfraum case, the DANTE temperature is $193 \mathrm{eV}$ and the sample temperature is $186 \mathrm{eV}$ (difference of $16 \%$ in flux). All temperatures are quoted for simulation time $t=1.0 \mathrm{~ns}$. So, the presence of the disk that divides the Hohlraum in half affects the DANTE-measured drive by about twice as much as it affects the sample drive. By comparing Fig. 10 with Fig. 1(c) it is clear that the proportionally larger drop in DANTE temperature in the halfraum case is due to the fact that in a Hohlraum, DANTE sees some of the laser hot-spot emission from the far side of the Hohlraum, which is caused by the beams entering through the far LEH. In the halfraum, DANTE sees instead wall reemission from the far end of the halfraum. In situations where low-angle beams, which cross the Hohlraum midplane, are used, one sees similar trends, with the only difference being that the low-angle beams hit the back wall rather than the side wall

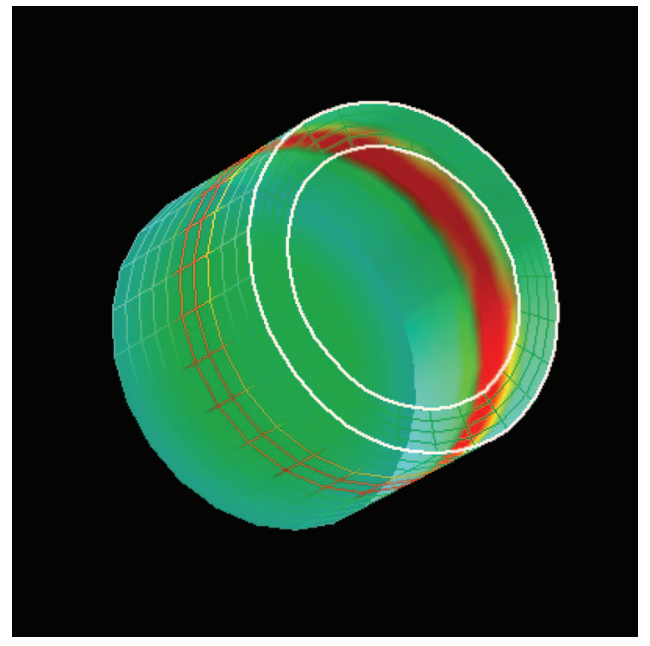

FIG. 10. (Color) The DANTE view at $t=1.0 \mathrm{~ns}$ of a halfraum simulation, created by dividing the Hohlraum shown in Fig. 1 in half with a gold disk. Note that from this viewing angle, some of the laser hot spots on the far side of the Hohlraum, caused by beams entering the Hohlraum through the far LEH, are visible [in Fig. 1(c)], which is, of course, not the case with the dividing disk present (as in this figure).

of the halfraum. Such considerations are relevant to NIF (Ref. 15) configurations, where there are a significant number of midplane-crossing low-angle beams.

\section{BEAM POINTING WITHIN A HALFRAUM}

One straightforward way to try to control the drive properties in a halfraum is to adjust the beam pointing. Here we explore the dependence of the drive onto a sample mounted on the back wall of a halfraum as the beam pointing varies. We also monitor the DANTE temperature as a function of beam pointing, from the usual $\mathrm{LEH}$ view with a $37.4^{\circ}$ angle to the halfraum axis. To simplify the situation, we revert to the pointing used in the experiments of Decker et al. ${ }^{4}$ (the single ring pointing in which all 15 beams make a single ring of hot spots) and our initial modeling in Sec. II. The other halfraum properties are the same as those we have used for the previous modeling: variable XCE and albedo as described earlier, a halfraum length of $1150 \mu \mathrm{m}$ and a diameter of $1600 \mu \mathrm{m}$, and a LEH diameter of $1200 \mu \mathrm{m}$. All 15 beams are taken to have perfect square profiles over $1 \mathrm{~ns}$ and total energies of $500 \mathrm{~J} /$ beam. In all the simulations presented in this section, the beams are focused at the point at which they cross the halfraum axis. We make an exception for several beams in the simulation with the deepest beam pointing, where we had to pull back the focus position slightly in order to keep the beams from clipping the LEH lip.

We present four simulations, depicted in Figs. 10 and Fig. 11, in which the beam pointing varies by $150 \mu \mathrm{m}$ for each simulation. The simulation already discussed and shown in Fig. 10, with the beam pointing at the LEH plane, corresponds to the default pointing used in the previous sections, with a hot-spot distance of $480 \mu \mathrm{m}$ from the LEH plane. The three simulations shown in Fig. 11 are for our shallower pointing and two deeper pointings. 

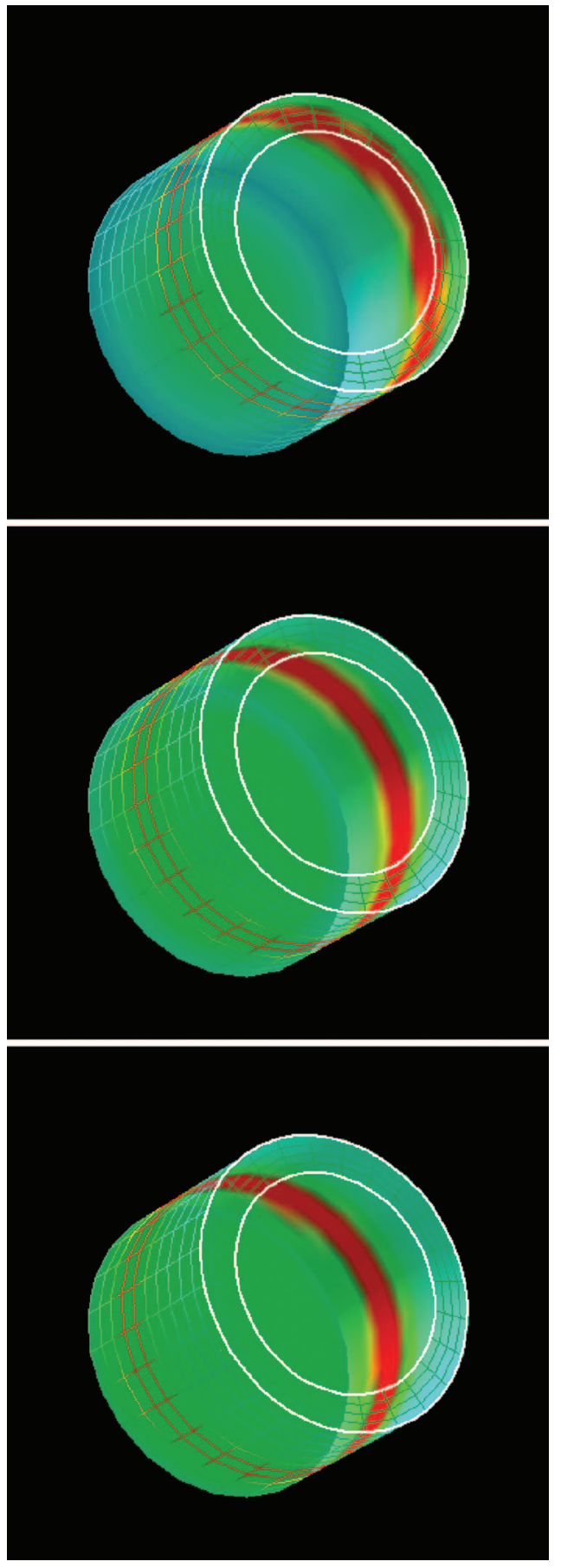

FIG. 11. (Color) Series of halfraum simulations in which the beam pointing was varied. The images show the DANTE view at $t=1.0 \mathrm{~ns}$. The top panel has the beam pointing pulled back $150 \mu \mathrm{m}$ from the nominal position, shown in Fig. 10. Recall that "nominal" means that the cone 3 beams cross the halfraum axis at the LEH plane and the cone 2 beams cross the axis $400 \mu \mathrm{m}$ outside the LEH. The middle panel has the beam pointing $150 \mu \mathrm{m}$ farther into the halfraum than the nominal case, and the bottom panel has the pointing $300 \mu \mathrm{m}$ farther than the nominal case.

In Fig. 12 we show the trend of radiation temperature on the back-wall sample along with the DANTE temperature as a function of beam pointing. They are similar to what is seen in Hohlraums (see Fig. 5) where deeper pointings generate higher temperatures by decreasing LEH losses and positioning hot spots more favorably. The flattening out of the

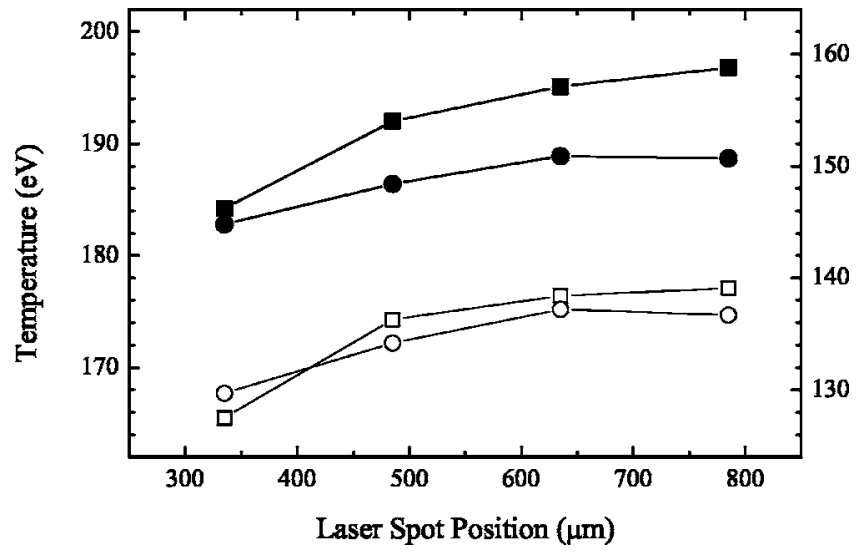

FIG. 12. The radiation temperature on a sample mounted on the end of a halfraum (circles) and measured by DANTE (squares) for the four different beam pointings shown in Figs. 10 and 11 at two different simulation times: $t=1.0 \mathrm{~ns}$ (filled symbols; left-hand axis) and $t=100 \mathrm{ps}$ (open symbols; righthand axis). The mean laser spot position is measured with respect to the LEH plane, so deeper pointings are further right.

sample temperature trend with the deepest pointing is due to the higher obliquity of the hot spots as seen by the sample. We note that, as detailed in the previous section, the DANTE temperature exceeds the radiation temperature onto the sample by between 5 and $10 \mathrm{eV}$ at late times when the halfraum albedo is high. But at earlier times, when the albedo is much lower, the two temperatures are more similar. When the albedo is low, the distinction between weak wall reemission and cold LEH is minimal relative to the hot spots, so the sample seeing wall plus LEH and DANTE seeing solely wall give similar radiation temperatures.

It is clear from these beam pointing simulations, and especially from inspecting Fig. 11, that the LEH lip can play an important role, as it affects the DANTE view factors in addition to the effect it has on keeping reemitted radiation from escaping out of the LEH. To investigate this quantitatively, we have repeated the four view-factor simulations shown in Fig. 11, but without the LEH lip.

The results of this series of simulations are shown in Fig. 13 , where the trends of sample and DANTE radiation temperature are displayed. The removal of the LEH lip has several effects. The DANTE temperatures are lower and some-

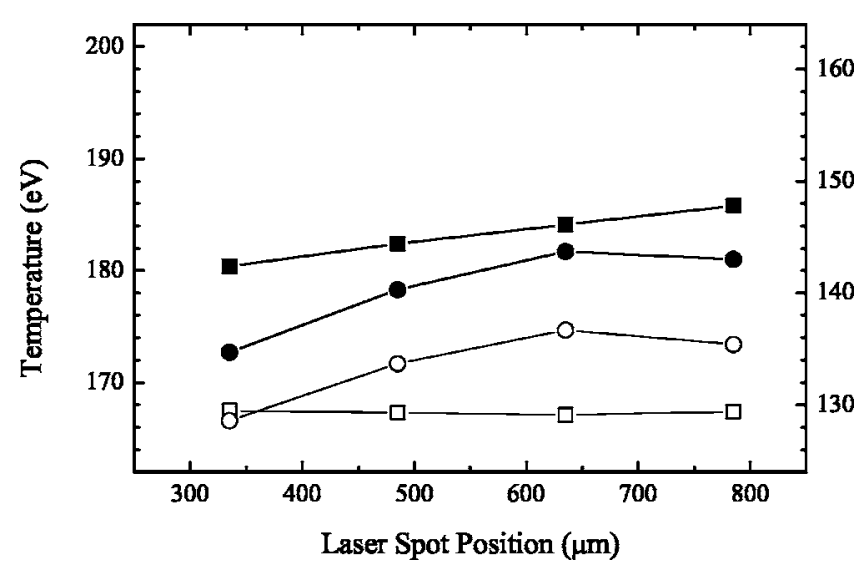

FIG. 13. Same as Fig. 12, but with no LEH lip (i.e., 100\% LEH). 
what less dependent on the beam pointing. This appears to be because the portion of the halfraum wall nearest the LEH is much colder in these simulations than in those with a LEH lip. In the simulations with the lip (i.e., three-quarter LEH), the DANTE temperature increases more dramatically as the pointing becomes deeper because without the LEH lip, the shallower beam pointings have larger LEH losses than in the case with the LEH lip. For the deeper pointings, the relatively cool wall near the LEH effectively dilutes the contributions from the hot spots and wall reemission from farther in the halfraum.

The sample radiation temperature is somewhat lower (by as much as $10 \mathrm{eV}$ ) in these simulations without the lip compared to those with the LEH lip, due to increased losses through the LEH. The trend of increased sample temperature with deeper pointing is apparent in these simulations without the LEH lip, as it was in the simulations with the lip. The trend is somewhat stronger in the simulations without the lip, likely because the increased LEH losses are stronger for the shallower pointing cases. At early times, however, the sample temperature is not strongly affected by the presence or absence of the lip, as the distinction between wall reemission and cold LEH is not very great when the albedo is low and the drive onto the sample is dominated by direct emission from the hot spots. In summary, the sample temperature drops more than the DANTE temperature due to the absence of the LEH lip (at least at later times) because the sample sees a bigger cold LEH.

We performed a final series of simulations with varying beam pointing, but this time with a more natural pointing configuration, in which the aim points of both cones 2 and 3 are the same, causing two separate rings of hot spots on the walls of the halfraum. We also revert back to the standard, three-quarter LEH for this series. The nominal pointing in this case has all 15 beams pointed (and focused) at the LEH center. This makes a ring of hot spots (from cone 3) at $480 \mu \mathrm{m}$ from the LEH plane and another ring (from cone 2) at $890 \mu \mathrm{m}$ from the LEH plane. The weighted mean pointing of ten beams at $480 \mu \mathrm{m}$ and five beams at $890 \mu \mathrm{m}$ is $617 \mu \mathrm{m}$. As in the previous two sets of simulations, we vary the pointing by moving all the beams inward by $150 \mu \mathrm{m}$ and then by $300 \mu \mathrm{m}$, and also calculate a case in which all the beams are pulled out $150 \mu \mathrm{m}$ from this nominal pointing.

The results of this series of simulations are summarized in Fig. 14. The general trends shown previously are also seen in this series of calculations. The drive temperature onto the sample is relatively independent of pointing, except for the most extreme cases, in which it is a little cooler. This is because the effect of the obliquity of the hot-spot view is even more extreme, with the cone 2 beams pointed further into the halfraum. The DANTE temperatures are also quite independent of beam pointing and modestly higher than the sample radiation temperatures (more so at the later times, when the wall albedo is higher).

\section{OPTIMIZING HALFRAUM GEOMETRY}

The results from the previous section can be used to maximize the radiation drive onto a sample mounted on the

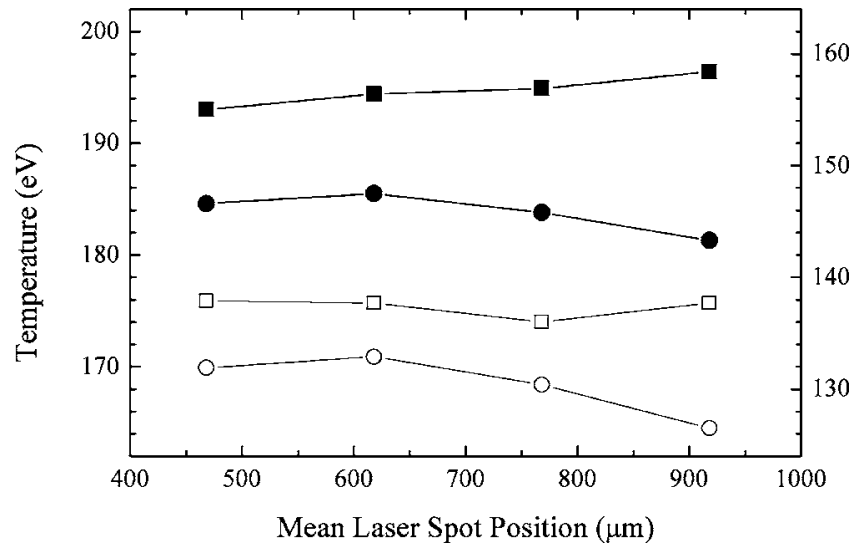

FIG. 14. Same as Fig. 12, but with the two beam cones making two different rings of hot spots, and using the three-quarter LEH.

back wall of a halfraum, as well as to relate radiation diagnostics from DANTE to the sample drive properties. In this section, we investigate the dependence of drive properties on the halfraum length and also on the presence of internal LEH shields as well as a foil just outside the LEH. For simplicity, we keep the halfraum diameter $(1600 \mu \mathrm{m})$ and LEH diameter $(1200 \mu \mathrm{m})$ the same for these simulations and also do not vary the beam properties. In this first set of simulations, the beam pointing is always at the LEH center (with the beams all focused at this point as well). Thus, as the halfraum length changes, the distance of the hot spots from the sample also changes.

We performed a series of four simulations in which the halfraum length is varied from 1000 to $1450 \mu \mathrm{m}$ in steps of $150 \mu \mathrm{m}$. In Fig. 15, in the top panel, we plot DANTE and sample temperatures at two different simulation times as a function of halfraum length. These temperatures are relatively independent of length, with only a slight decrease in radiation temperature for the longer halfraums. The tendency toward lower temperatures due to the greater wall area in the longer halfraums is partially offset by fewer radiation losses out of the LEH, and for the sample, the smaller LEH solid angle as the halfraum lengthens also tends to offset the increased wall losses. The drop in the sample radiation temperature for the shortest halfraums is due to the obliquity of the hot spots as seen from the sample, especially the cone 2 spots.

To investigate whether the slight decrease in the radiation temperature of the back-wall-mounted sample is primarily due to its distance from the hot spots, we repeated the previous series of simulations, but with the beam pointing adjusted in each case to keep the hot spots' distance from the sample constant as the halfraum length was adjusted. For this series of calculations, we kept the nominal (LEH-centered) pointing for the standard halfraum length of $1150 \mu \mathrm{m}$. However, for each of the other three halfraum lengths, we moved all 15 beams either in or out according to the halfraum length so that the pointings and thus the hot spots' positions were always at the same distance from the sample. Thus, for the 1000- $\mu \mathrm{m}$-long halfraum, the beam pointing was $150 \mu \mathrm{m}$ outside of the LEH, at the halfraum axis. For the $1300 \mu \mathrm{m}$ halfraum, the pointing was $150 \mu \mathrm{m}$ inside the LEH, and for 

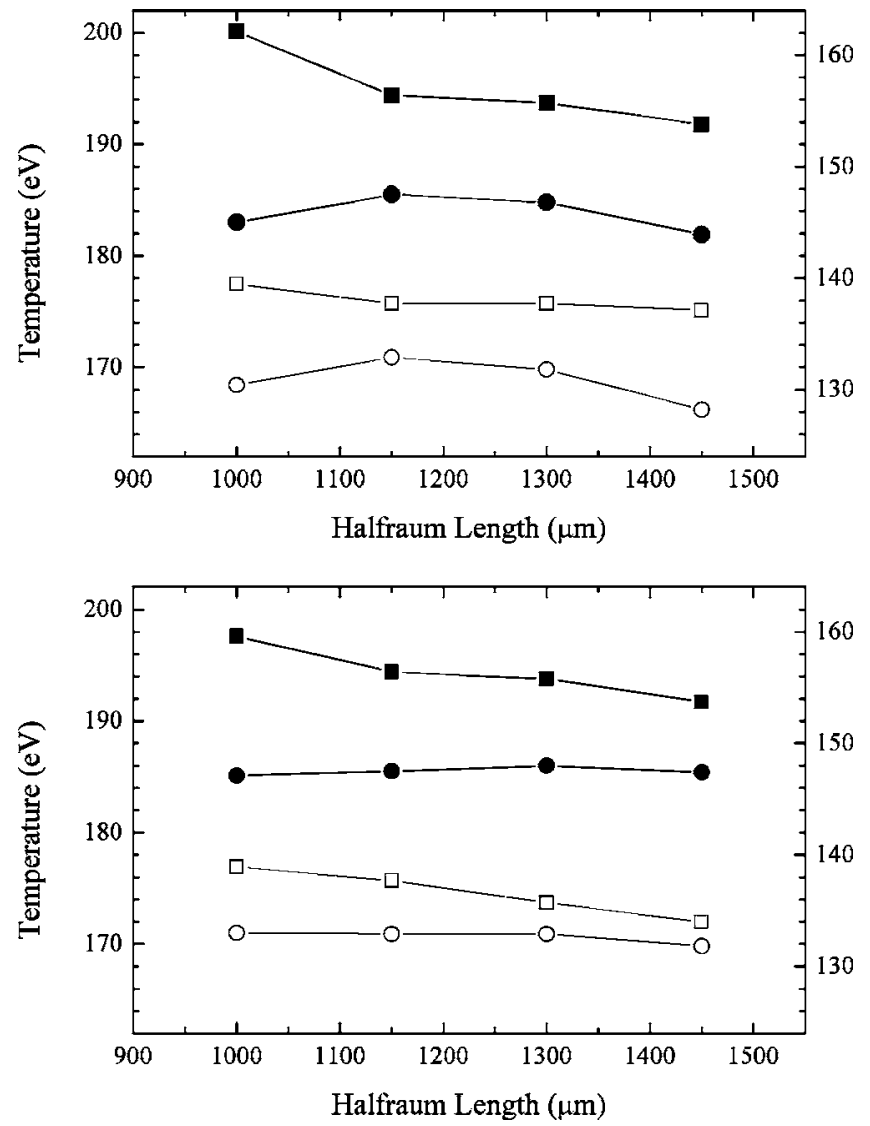

FIG. 15. Temperature as a function of halfraum length for four simulations having identical beam pointings with respect to the LEH [nominal beam pointing regardless of halfraum length (top)] and with the beam pointing varying according to halfraum length (bottom). The solid symbols are from a simulation time of $t=1.0 \mathrm{~ns}$ (left-hand axis) while the open symbols are from $t=100 \mathrm{ps}$ (right-hand axis). The squares are DANTE temperatures and the circles are sample radiation temperatures.

the $1450 \mu \mathrm{m}$ halfraum, it was $300 \mu \mathrm{m}$ inside. For all but the longest halfraum, all the beams were focused at the pointing position. For the longest halfraum, we had to focus the beams closer to the LEH plane, to prevent beam clipping on the LEH lip.

The results of this series of simulations are shown in the bottom panel of Fig. 15. The primary result is that the sample temperature is almost totally independent of halfraum length (at both $100 \mathrm{ps}$ and $1 \mathrm{~ns}$ ). This is, of course, counter to the expectations of standard Hohlraum temperature power balance analysis, which would predict lower temperatures as the halfraum was lengthened (as is seen in the first set of simulations discussed in this section). Clearly, the fact that the sample's view factor of hot spots is the same in each of these four cases (because the pointing is constant relative to the sample itself) is much more important than the addition of a extra wall area as the halfraum is lengthened. Furthermore, the LEH subtends a smaller solid angle as seen from the location of the hot spots in the longer halfraums, so LEH losses are minimized, even as wall losses increase. The DANTE temperature decreases somewhat with an increasing halfraum length because the wall in the DANTE field of view includes contributions from regions farther from the hot spots when the halfraum is longer, and these wall regions are
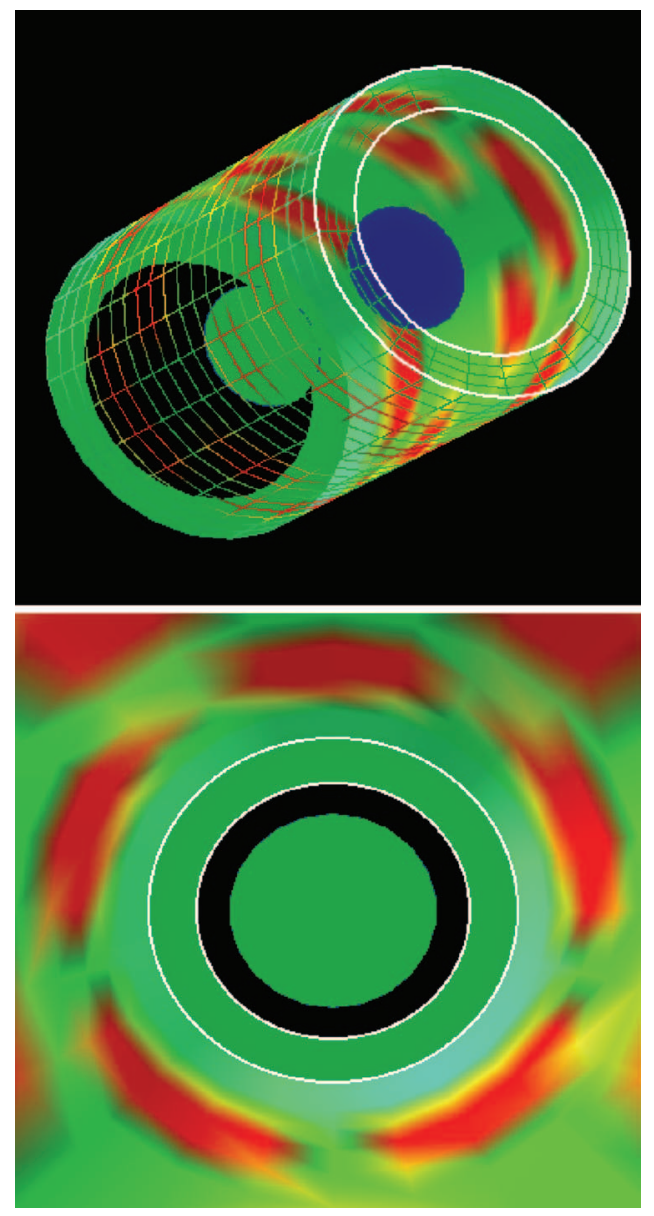

FIG. 16. (Color) Emission temperature color maps, at $t=1 \mathrm{~ns}$, of the Hohlraum simulation with the LEH shields (compare to the otherwise identical simulation shown in the top panel of Fig. 5, but without the LEH shields). The top panel shows the usual DANTE view, while the bottom panel shows a view from near the Hohlraum midplane, looking toward one of the LEHs. Note that the shield subtends an angle almost as large as the $\mathrm{LEH}$, as seen from the midplane, and that it is about as hot as the inside of the LEH lip.

colder, as there is a negative axial temperature gradient associated with the hot spots.

In order to maximize the radiation drive onto a sample, or generally in a Hohlraum or halfraum, extra walls or barriers or other complex geometries can be employed. Boosts of the drive onto a capsule have been demonstrated via the use of walls on the interior of Hohlraums that block the capsule's view of the LEH. ${ }^{16}$

We performed a simulation to investigate the effects of such "LEH shields." Again using a standard size (2300 $\times 1600 \mu \mathrm{m})$ Hohlraum with three-quarter LEHs, we put two $600-\mu \mathrm{m}$-diam gold disks centered on the Hohlraum axis $600 \mu \mathrm{m}$ from the Hohlraum midplane. We used the nominal beam pointing, with all 15 beams on each side of the Hohlraum pointed and focused at the center of the LEH. As expected, and shown experimentally, these LEH shields boost the temperature at the midplane of the Hohlraum by blocking much (some) of the LEHs seen by a sample (hot spots), decreasing the effective view factor of cold LEH and preventing some of the radiation losses out of the LEH. The usual emission temperature color maps from two different 

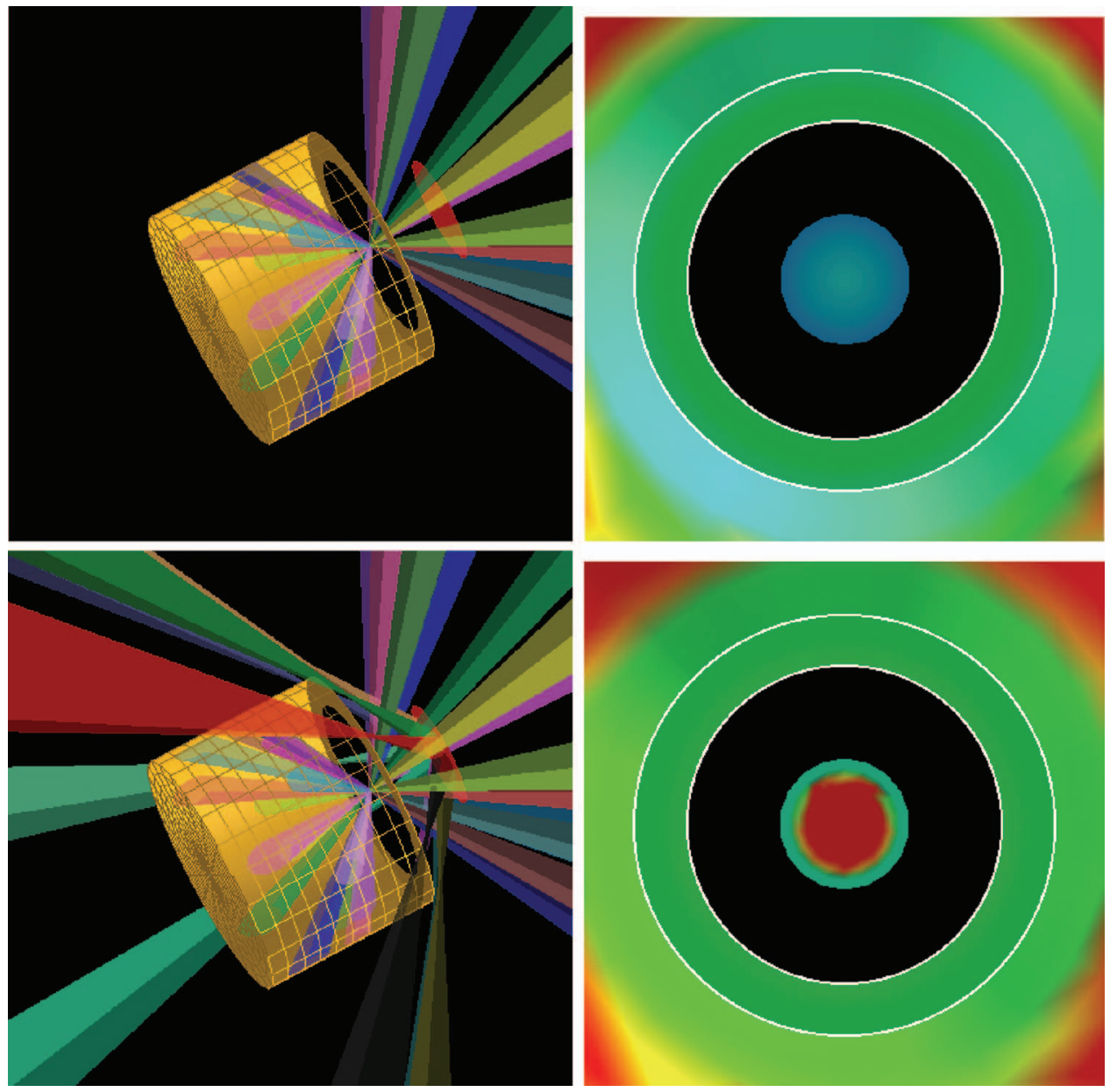

FIG. 17. (Color) Two halfraum simulations with a metal foil just outside the LEH. In the simulation shown in the top two panels (DANTE view on the left and view from the sample position on the right), there are no beams onto the foil. The foil simply acts to absorb and reemit radiation that exits through the LEH. In the bottom two panels, there are ten beams onto the foil, which significantly increase the radiation flux inside the halfraum. The snapshots in the right-hand column show emission temperatures at $t=1.0 \mathrm{~ns}$. views are shown for this simulation in Fig. 16.

We monitored the radiation temperature in this simulation on a sample at the center of the Hohlraum (and facing one of the LEHs) as well as on a midplane wall-mounted sample. The radiation temperature on these two samples never varied by more than $2 \mathrm{eV}(135 \mathrm{eV}$ vs $135 \mathrm{eV}$ at $t=100 \mathrm{ps}$ and $197 \mathrm{eV}$ vs $195 \mathrm{eV}$ at $t=1 \mathrm{~ns})$. In the simulation without the shields, the sample temperatures are nearly identical at early times (134 and $135 \mathrm{eV}$ at $t=100 \mathrm{ps}$ for the Hohlraum-center and wall-mounted samples, respectively) but are somewhat lower at later times $(190$ and $193 \mathrm{eV}$ at $t=1 \mathrm{~ns})$. The lack of effect of the LEH shields on the sample temperature at early times is due to the fact that the shields themselves are not yet hot enough to emit significant radiation; they are nearly as cold as the LEHs that they block. But at late times, the shields boost the temperature on a sample at the center of the Hohlraum by $7 \mathrm{eV}$, corresponding to $16 \%$ in flux. As expected, the boost at the midplane wall is significantly smaller ( $2 \mathrm{eV}$, or $4 \%$ in flux) as it has the same unobstructed view of the LEH with or without the shields.

The DANTE temperature is significantly lower in the simulation with the shields $(197 \mathrm{eV}$ at $t=1 \mathrm{~ns}$ vs $207 \mathrm{eV}$ at the same simulation time in the case without the shields). As can clearly be seen in the simulation (Fig. 16), the lower DANTE temperature is due the fact that the back of the shield is in the DANTE view, blocking this diagnostic's view of some hot spots and wall reemission. We note that, at least in this particular simulation, the radiation temperature on a sample at the Hohlraum center and the DANTE temperature are identical.

A strategy similar to the use of LEH shields might involve putting metal foils outside the LEH to absorb radiation lost out of the LEH and reemit it back into the Hohlraum or halfraum. In Fig. 17 we show an example of this scheme, in which a circular foil with a diameter of $700 \mu \mathrm{m}$ is hung $500 \mu \mathrm{m}$ outside the LEH. One potential advantage is that a foil on the exterior can be irradiated with unused beams from the other (non-LEH-facing) side of the target chamber to provide an additional source of $x$ rays to heat the halfraum.

We performed two simulations of the halfraum with the foil in the configuration described above, and using the nominal pointing (all 15 beams pointed at the center of the LEH plane) and halfraum size $(l=1150 \mu \mathrm{m})$. In one, we do not irradiate the foil at all, and in the other, we irradiate the foil with all ten cone 3 beams from the other side of the halfraum, using the same power profile as the halfraum beams (1 ns square pulses with $500 \mathrm{~J} / \mathrm{beam}$ ). This additional source of radiation makes the entire halfraum hotter. In Fig. 18 we compare the spectra incident on the sample (mounted as usual on the center of the back wall of the halfraum) from the two cases with the foil (irradiated and not) to the standard case without the foil. It can be seen from this figure that while simply adding the foil makes a very little difference (sample radiation temperature of $187 \mathrm{eV}$ vs $185.5 \mathrm{eV}$, or $3 \%$ 


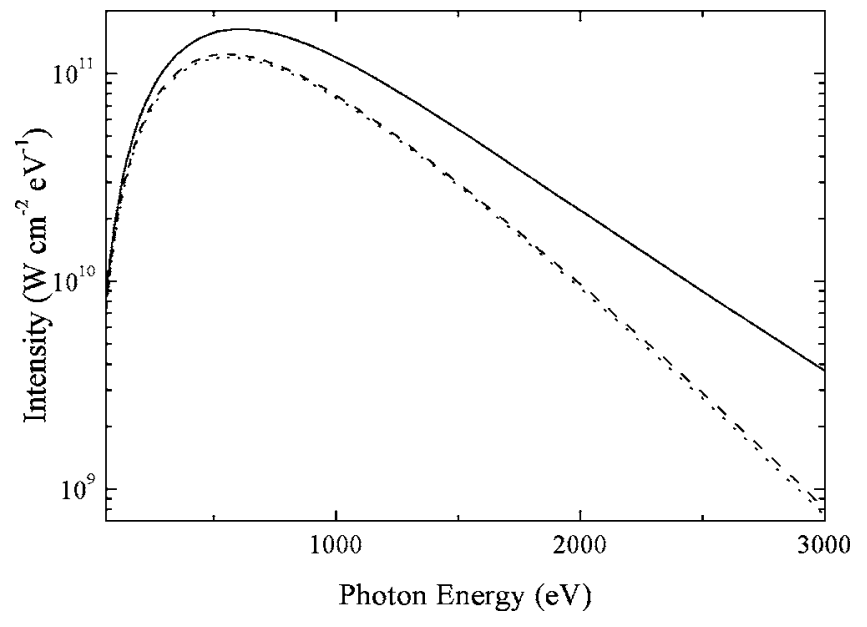

FIG. 18. Comparison of spectra incident on the center of the sample in our standard $(l=1150 \mu \mathrm{m})$ halfraum at $t=1.0 \mathrm{~ns}$, with the standard beam pointing. The dotted line represents the simulation with no foil, the dashed line (nearly coincident with the dotted line) represents the simulation with a foil outside the LEH, and the solid line is from the simulation with the foil heated by ten beams.

in flux), irradiating the foil makes a large difference, raising the radiation temperature on the sample to $207 \mathrm{eV}$ (representing a 55\% increase in total flux) and roughly doubling the radiation flux at $2 \mathrm{keV}$.

\section{CONCLUSIONS}

We have presented results from numerical view-factor simulations performed to investigate the variation of radiation conditions as a function of spatial, geometrical, and beam pointing conditions for Hohlraums and halfraums at OMEGA. In addition, we have presented results showing sometimes significant differences in the Hohlraum wall temperatures viewed by DANTE and the radiation drive temperatures seen by experimental packages attached to the Hohlraum or halfraum walls. Because the radiative flux and thus the heating scales as the fourth power of the temperature, even modest differences in wall temperatures can be significant. View-factor simulations, such as those presented here, provide a means of simulating Hohlraum radiation characteristics and interpreting wall emission measurements (e.g., DANTE), and can be of value in designing and interpreting experiments at OMEGA and future experiments at NIF.

We find, specifically, from several series of simulations, that the radiation drive onto a sample can differ substantially from that measured by an absolutely calibrated x-ray detector, such as DANTE, even when the diagnostic line of sight is through a LEH. This is especially true in Hohlraums, as compared to halfraums, and at late times (when wall albedos are high). In the standard Hohlraum simulations we carried out, the radiation temperature on a sample at the Hohlraum midplane is roughly $15 \mathrm{eV}$ lower than the DANTE temperature. In standard halfraum configurations, there is a good agreement between DANTE temperatures and radiation temperatures onto a sample mounted at the center of the back wall (roughly a $5 \mathrm{eV}$ discrepancy at $200 \mathrm{eV}$ ). This better agreement is primarily due to the fact that in a Hohlraum, the DANTE temperature is boosted with respect to a halfraum because DANTE sees some of the hot spots on the far side of the Hohlraum, from beams entering from the opposite side. We also find that midplane radiation temperatures in Hohlraums are very similar to radiation temperatures onto a sample suspended at the center of a Hohlraum, and further, that the orientation of such a sample has very little effect on the radiation temperature.

It was also shown that even when radiation temperatures between two different samples, or between a sample and DANTE, are very similar, the respective spectral energy distributions can differ significantly. The primary trend we found is that incident spectra are harder than the equivalent Planckian spectra having the same radiation temperature. Another milder trend is that the spectrum onto a sample tends to be harder than that seen by DANTE.

Variations in beam pointing and halfraum length were found to have a relatively little effect, generally, on either the sample radiation temperature or the DANTE temperature, except in extreme cases. The mean laser spot position can be varied anywhere from roughly 400 to $800 \mu \mathrm{m}$ from the LEH plane in a standard halfraum without changing either the DANTE temperature or the drive temperature onto a sample more than a few eV. A 1600- $\mu$ m-diam halfraum will provide maximal drive temperatures with nominal beam pointing when the halfraum has a length anywhere between 1100 and $1400 \mu \mathrm{m}$. However, the size of the LEH can have a significant effect on both DANTE and sample temperatures. Furthermore, the presence of a capsule in a Hohlraum will lower both the DANTE temperature and the sample temperature in a predictable way due to increased losses into the low-albedo capsule. Finally, the drive onto a sample can be increased significantly by the inclusion of LEH shields (which also have the effect of lowering the DANTE temperature, bringing it into much closer agreement with the sample temperature) or by irradiating a foil placed just outside the LEH of a halfraum.

\section{ACKNOWLEDGMENTS}

We thank Rick Olson for useful discussions, Dave Conners for performing some initial calculations, and Micah Walter-Range for his help producing some of the images.

We are grateful for support through Grant No. DEFG03-OOSF22023 (from the DOE/NLUF program) to Prism Computational Sciences, and Research Corporation Grant No. CC5489 to Swarthmore College.

${ }^{1}$ J. D. Lindl, P. Amendt, R. L. Berger, S. G. Glendinning, S. H. Glenzer, S. W. Haan, R. L. Kauffman, O. L. Landen, and L. J. Suter, Phys. Plasmas 11, 339 (2004).

${ }^{2}$ M. D. Rosen, Phys. Plasmas 3, 1803 (1996).

${ }^{3}$ H. N. Kornblum, R. L. Kauffman, and J. A. Smith, Rev. Sci. Instrum. 57, 2179 (1986).

${ }^{4}$ C. Decker, R. E. Turner, O. L. Landen, L. J. Suter, P. Amendt, H. N. Kornblum, B. A. Hammel, T. J. Murphy, J. Wallace, N. D. Delamater et al., Phys. Rev. Lett. 79, 1491 (1997).

${ }^{5}$ T. R. Boehly, D. L. Brown, R. S. Craxton, R. L. Keck, J. P. Knauer, J. H. Kelly, T. J. Kessler, S. A. Kumpan, S. J. Loucks, S. A. Letzring et al., Opt. Commun. 133, 495 (1997).

${ }^{6}$ J. J. MacFarlane, J. Quant. Spectrosc. Radiat. Transf. 81, 287 (2003). 
${ }^{7}$ J. J. MacFarlane, I. Golovkin, and P. R. Woodruff, "Inertial Fusion Sciences and Application 2003," edited by B. A. Hammel, D. D. Meyerhofer, J. Meyer-ter-Vehn, H. Azechi (American Nuclear Society, 2004), p. 166. ${ }^{8}$ J. D. Schnittman and R. S. Craxton, Phys. Plasmas 7, 2964 (2000).

${ }^{9}$ L. J. Suter, R. L. Kauffman, C. B. Darrow, A. A. Hauer, H. Kornblum, O. L. Landen, T. J. Orzechowski, D. W. Phillion, J. L. Porter, L. V. Powers et al., Phys. Plasmas 3, 2057 (1996).

${ }^{10}$ W. A. Stygar, R. E. Olson, R. B. Spielman, and R. J. Leeper, Phys. Rev. E 64, 026410 (2001).

${ }^{11}$ R. E. Olson, R. J. Leeper, S. C. Dropinski, L. P. Mix, G. A. Rochau, S. H. Glenzer, O. S. Jones, L. J. Suter, J. L. Kaae, C. H. Shearer et al., Rev. Sci. Instrum. 74, 2186 (2003).
${ }^{12}$ M. Murakami and J. M. Meyer-ter-Vehn, Nucl. Fusion 31, 1315 (1991).

${ }^{13}$ C. A. Back, J. D. Bauer, J. H. Hammer, B. F. Lasinski, R. E. Turner, P. W. Rambo, O. L. Landen, L. J. Suter, M. D. Rosen, and W. W. Hsing, Phys. Plasmas 7, 2126 (2000).

${ }^{14}$ D. H. Cohen, J. J. MacFarlane, P. Jaanimagi, O. L. Landen, D. A. Haynes, D. S. Conners, K. L. Penrose, and N. C. Shupe, Phys. Plasmas 11, 2702 (2004).

${ }^{15}$ J. A. Paisner, J. D. Boyes, S. A. Kumpan, W. H. Lowdermilk, and M. S. Sorem, Laser Focus World 30, 75 (1994).

${ }^{16}$ P. Amendt, S. G. Gendinning, B. A. Hammel, O. Landen, and L. J. Suter, Phys. Rev. Lett. 77, 3815 (1996). 